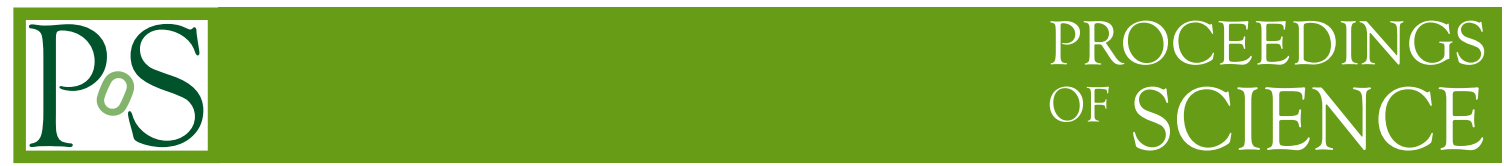

\title{
Searches for resonant di-boson production at CMS
}

\section{Dennis Roy* on behalf of the CMS Collaboration}

RWTH Aachen University, III. Physics Institute B

E-mail: dennis.royecern.ch

Most recent CMS results on searches for heavy resonances decaying into gauge and Higgs bosons (VV where $\mathrm{V}=\mathrm{W}, \mathrm{Z}, \mathrm{H}$ ) are presented. The results are based on the large dataset collected during Run 2 of the LHC at a centre-of-mass energy of $13 \mathrm{TeV}$. The analyses are optimised for high sensitivity over a large range in resonance mass. Jet substructure techniques are used to identify hadronic decays of highly-boosted $\mathrm{W}, \mathrm{Z}$, and $\mathrm{H}$ bosons.

European Physical Society Conference on High Energy Physics - EPS-HEP2019 -

10-17 July, 2019

Ghent, Belgium

${ }^{*}$ Speaker. 


\section{Introduction}

At the time of this talk there exist 16 analyses by CMS [1] highlighting resonant di-boson production at high masses using data from 2016 onwards $[2,3,4,5,6,7,8,9,10,11,12,13$, $14,15,16,17]$. These high mass resonances can be interpreted as new particles predicted by beyond-the-SM theories. These include theories about an extended Higgs-like EW sector, a TwoHiggs-Doublet Model (2HDM) or Minimal Supersymmetric SM (MSSM), a Bulk Graviton or a heavy vector triplet (HVT).

\section{Hadronic jet reconstruction}

Bosons which originate from the decay of a high mass resonance have a large Lorentz boost. This means that its decay products are collimated. If the boson decays into a pair of jets, the collimated jets can then be reconstructed as a single "AK8" jet, which means that the Anti-kT algorithm [19] is used with a jet-cone size of $R=0.8$. This approach is employed by all analyses reconstructing hadronically decaying bosons from high mass resonances.

Analyses which search for resonances in a mass range which extends to low values of the mass (from the SM Higgs boson mass up to about $500 \mathrm{GeV}$ ) also still consider the hadronic decay into two low $p_{T}$ AK4 jets. These analyses split up their categorization between events where the boson is reconstructed from an AK8 jet (Boosted/Merged category), and events where it is reconstructed from two AK4 jets (Resolved category).

The AK8 jets are groomed using soft-drop (modified mass-drop tagger) algorithm [20], removing further constituents from soft, large-angle radiation. An AK8 jet's mass is calculated from the four-momentum of groomed jet.

The N-subjettiness $\tau_{N}$ [21] is used as discriminating variable for jet substructure. Specifically $\tau_{21}=\tau_{2} / \tau_{1}$ gives a measure of how likely it is that a jet is composed out of two subjets.

\section{3. $\mathrm{X} \rightarrow \mathrm{WW} / \mathbf{Z Z}$}

The high mass $X \rightarrow$ WW analysis studies the di-leptonic (WW $\rightarrow 212 v$ ) and semi-leptonic (WW $\rightarrow$ lvqq) decay channels [2], while the high mass $\mathrm{X} \rightarrow \mathrm{ZZ}$ analysis studies decay channels with four charged leptons ( $\mathrm{ZZ} \rightarrow 4 \mathrm{l}$ ), two charged leptons and two neutrinos (WW $\rightarrow 212 v$ ) and also the semi-leptonic ( $\mathrm{ZZ} \rightarrow 212 \mathrm{q})$ decay channel [3]. Here, an overview of the semi-leptonic channels are given, as they are very similar for both the WW and ZZ analyses.

Categories exist for events being tagged as being produced by VBF. This is achieved by imposing a cut in the WW analysis, while in the ZZ analysis performs the categorization by using the angular distributions of the decay products as input for a matrix element likelihood analysis (MELA) $[22,23]$. Events not passing the VBF requirement in either analysis are then checked whether they pass a ggH-tagging or b-tagging requirement in the $\mathrm{WW}$ or $\mathrm{ZZ}$ analysis respectively. The ggHtagging is also performed using the angular distributions of the decay products as input for MELA. In the $\mathrm{ZZ}$ analysis, an event is considered to be b-tagged, if both (sub)jets from the $\mathrm{Z}$ boson are b-tagged. Events that do not satisfy any of these requirements end up in the untagged category. Each of the three categories in both analyses respectively is further divided into a Resolved and a 
Merged category, as explained earlier, as well as according to the flavor of the visible lepton in the final state $(\mathrm{e} / \mu)$.

The major background for the WW and $\mathrm{ZZ}$ analyses comes from $\mathrm{W}+\mathrm{Jets}$ and $\mathrm{Z}+\mathrm{Jets}$ events respectively. While the signal region is defined using a number of cuts, such as the requirement on the reconstructed mass of the hadronically decaying boson of $65 \mathrm{GeV}<m_{W, \text { had }}<105 \mathrm{GeV}$ and $70 \mathrm{GeV}<m_{Z \text {,had }}<105 \mathrm{GeV}$, a sideband region for the dominant background is defined by selecting events outside of this given mass range. In the WW analysis this range includes $[40,65] \mathrm{GeV}$ or $[105,250] \mathrm{GeV}$. The $\mathrm{W}+\mathrm{Jets}$ background shape is taken from simulation, while the normalization of this background is fitted to data using this sideband region. In the $\mathrm{ZZ}$ analysis the mass ranges for the sideband are $[40,70] \mathrm{GeV}$ and $[135,180] \mathrm{GeV}$. Here the Z+Jets background is determined by selecting the data event in the sideband region and subtracting all sub-dominant, non Z-Jets events. These events are then scaled by a signal-region over sideband-region ratio extracted from simulation.

As the analyses do not find an excess in data over the expectation, upper limit are given at 95\% CL on the cross section times branching fraction (fig. 1). The WW analysis also provides exclusion limit for various interpretations in MSSM and 2HDM scenarios (fig. 2) [24].

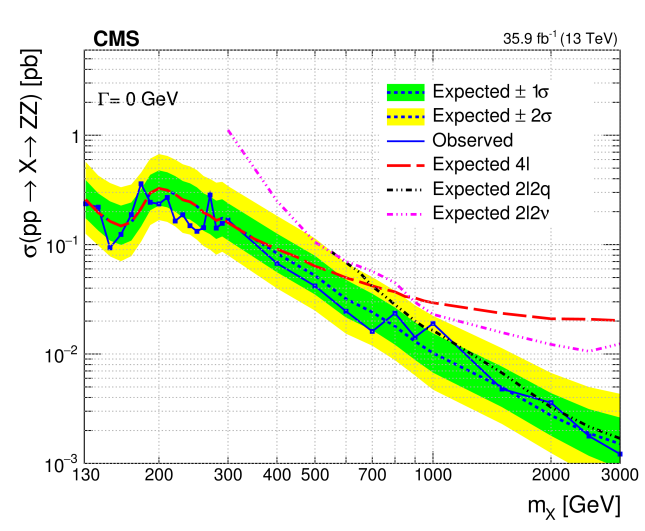

Figure 1: Expected and observed upper limits at the $95 \% \mathrm{CL}$ on the $\mathrm{pp} \rightarrow \mathrm{X} \rightarrow \mathrm{ZZ}$ cross section as a function of $m_{X}$ and for $\Gamma_{X}=0 \mathrm{GeV}$. [3]

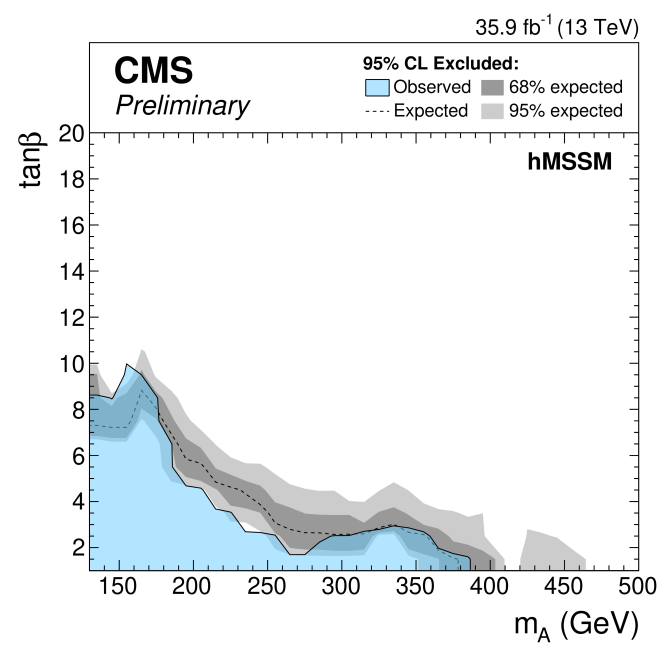

Figure 2: Expected and observed $95 \%$ CL upper limits on $\tan \beta$ as a function of $m_{A}$ for the hMSSM scenario. [2]

\section{4. $\mathbf{X} \rightarrow \mathbf{V V}$}

Other analyses searching for a high mass resonance from more general di-boson decays study semi-leptonic and/or full hadronic final states. Here the results of an all-hadronic analysis are presented [5].

This analysis makes use of the "designed decorrelated tagger" (DDT) method [25], as it was found that the N-subjettiness $\tau_{21}$ is correlated to the mass and the $p_{T}$ of the jets. This is illustrated for QCD background events in figure 3 as a function of $\rho^{\prime}=\log \left(m_{J}^{2} / p_{T}\right)$. Instead of $\tau_{21}$, a new variable $\tau_{21}^{D D T}=\tau_{21}-M \cdot \rho^{\prime}$ is defined, where $M$ is the correlation factor and is extracted directly from the 
slope in figure 3. The improvement of using $\tau_{21}^{D D T}$ instead of $\tau_{21}$ is seen in the ROC curved in figure 4 , which shows a significant decrease of the mistagging rate for the same tagging efficiency. The analysis is categorized into a high purity region $\left(\tau_{21}^{D D T}<0.43\right)$ and a low purity region $(0.43<$

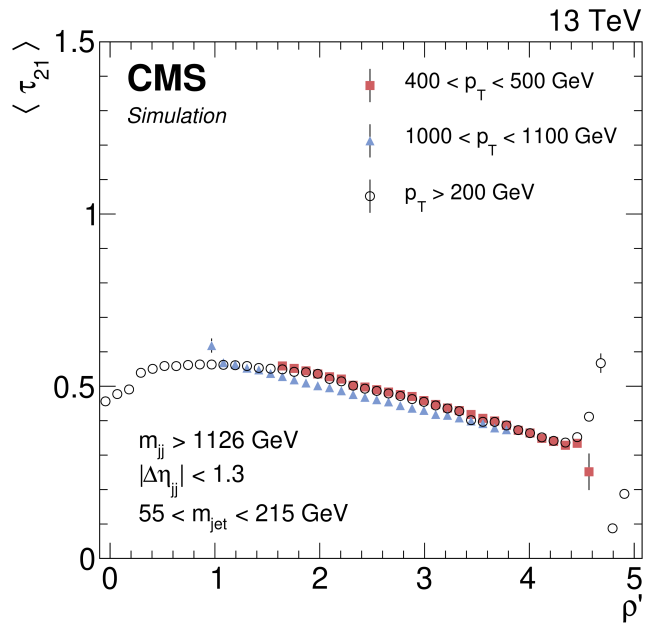

Figure 3: Distribution of the average $\tau_{21}$ over $\rho^{\prime}$ for QCD background events. [5]

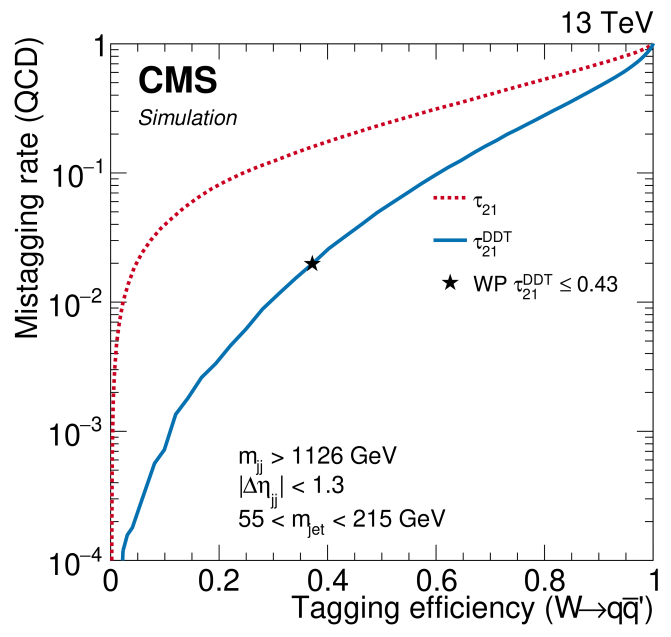

Figure 4: ROC curve of the mistagging rate over the tagging efficiency for $\tau_{21}$ (red, dotted) and $\tau_{21}^{D D T}$ (blue, solid). [5]

$\tau_{21}^{D D T}<0.79$ ). The event selection includes requirements on the transverse momentum of the jets of $p_{T}>200 \mathrm{GeV}$, as well as on the invariant di-jet mass of $m_{J J}>1126 \mathrm{GeV}$. The major background in this analysis comes from QCD multijet events.

There is no excess observed over the expectation. Upper limits on the cross section are provided for a Bulk graviton (fig. 5) and the HVT model B (fig. 6).

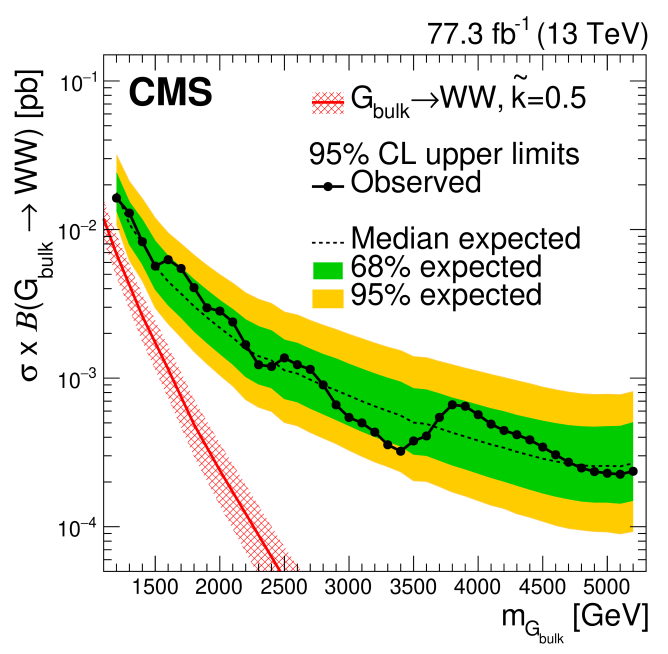

Figure 5: Observed and expected $95 \% \mathrm{CL}$ upper limits on the product of the production cross section and the branching fraction for the $G_{\text {bulk }} \rightarrow$ WW signal. [5]

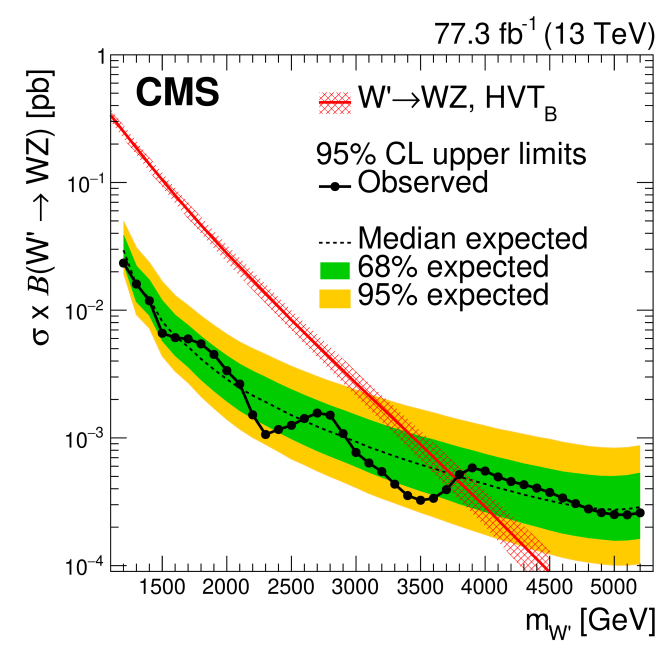

Figure 6: Observed and expected $95 \%$ CL upper limits on the product of the production cross section and the branching fraction for the $\mathrm{W}^{\prime} \rightarrow$ WZ signal. [5] 


\section{5. $\mathbf{X} \rightarrow \mathbf{H H} \rightarrow$ bbWW}

The results of an analysis studying the decay of $\mathrm{X} \rightarrow \mathrm{HH} \rightarrow \mathrm{bbWW}$ are presented, where the W bosons further decay semi-leptonically [9].

Categories are defined for the flavor of the visible lepton in the final state (electron or muon), on the working point of $\tau_{21}$ from the jets assigned to the hadronically decaying $\mathrm{W}$ boson, and on the $\mathrm{b}$-tagging working points of the two subjets from the $\mathrm{H}$ boson decaying into two $\mathrm{b}$ quarks. For the N-subjettiness there exists a high purity $\left(\tau_{21}<0.55\right)$ and a low purity $\left(0.55<\tau_{21}<0.75\right)$ region. For the subjet b-tagging there exists a loose category, which requires only one of the two subjets to pass the medium b-tagging working point, a medium category, where one subjets passes the medium b-tagging working point and the other subjet passes the loose b-tagging working point, and a tight category, where both subjets need to pass the medium b-tagging working point. Overall, 12 categories are defined with these definitions. Among the signal selection criteria there is the requirement of the scalar sum of the $p_{T}$ for all $\mathrm{AK} 4$ jets with at least $p_{T}>30 \mathrm{GeV}$ to be $H_{T}>400 \mathrm{GeV}$. The Drell-Yan background is suppressed by removing events in which a second lepton has a transverse momentum of $p_{T}<20 \mathrm{GeV}$. The major background in this analysis comes from $\mathrm{t} \overline{\mathrm{t}}$ events.

The backgrounds are determined by defining four categories. Events from all relevant background are sorted into these categories according to the number of generator-level quarks coming from the decay of the top quarks or $\mathrm{W}$ bosons. This is done because each of the background categories has a distinct shape in the distribution over $m_{b \bar{b}}$ (fig. 7). In each of the 12 signal categories, the yields of these four background categories, as well as the signal yield itself, are simultaneously estimated from data. No excess is observed over the expectation, and upper limits on the cross section are

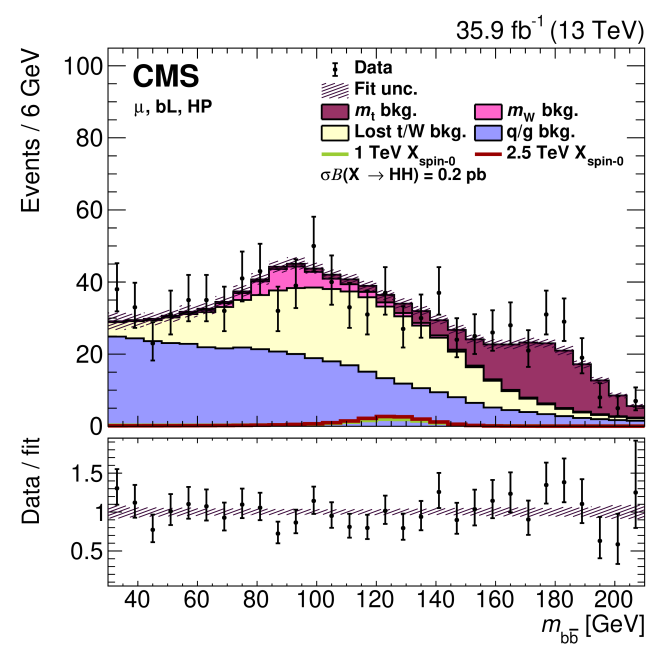

Figure 7: The fit result compared to data projected in $m_{b \bar{b}}$ for the $\mu$, Loose b-tagging, High Purity event category. The fit result is the filled histogram, with the different colors indicating different background categories. [9]

given for a spin 0 resonance (fig. 8) and a spin 2 resonance (fig. 9). 


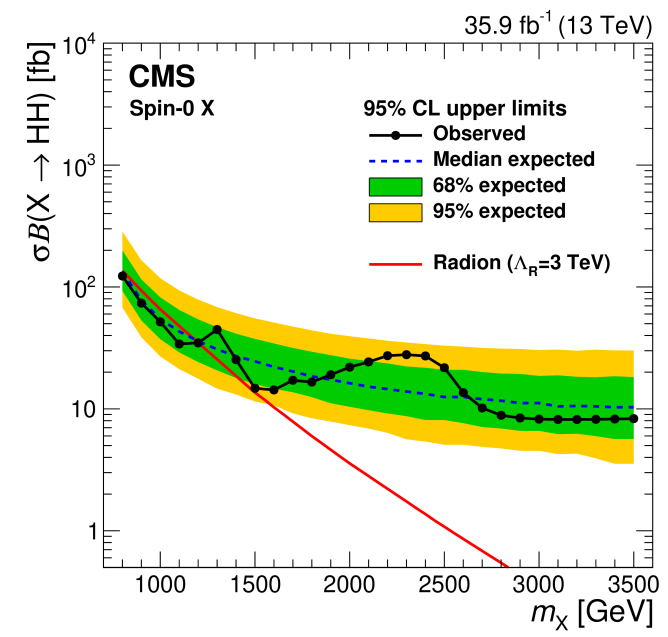

Figure 8: Observed and expected $95 \%$ CL upper limits on the product of the cross section and branching fraction to $\mathrm{HH}$ for a generic spin-0 boson. [9]

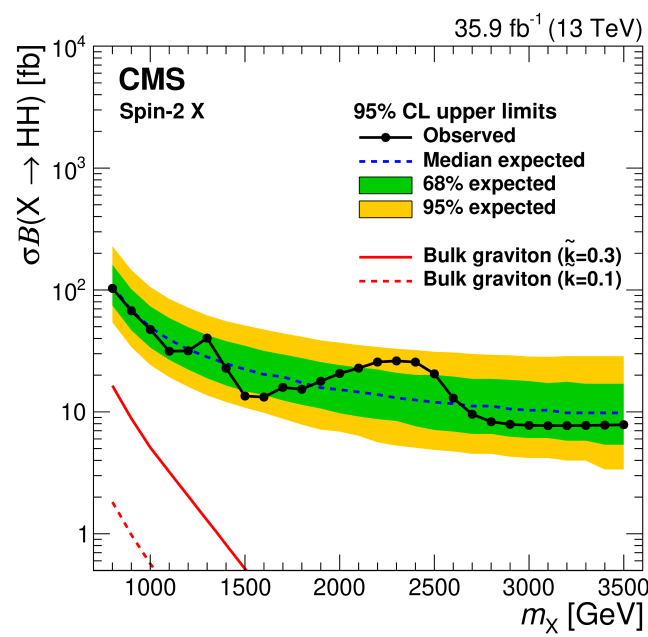

Figure 9: Observed and expected 95\% CL upper limits on the product of the cross section and branching fraction to $\mathrm{HH}$ for a generic spin-2 boson. [9]

\section{Conclusion}

Only a few analyses out of all 16 have been covered here. While there is no observation of a high mass resonance, the upper limits on signals have significantly improved with respect to those provided from Run 1 analyses. To give an example, the upper limit on the HVT model B using all di-boson analyses has improved to exclude a high mass resonance up to $4.5 \mathrm{GeV}$ (fig. 10).

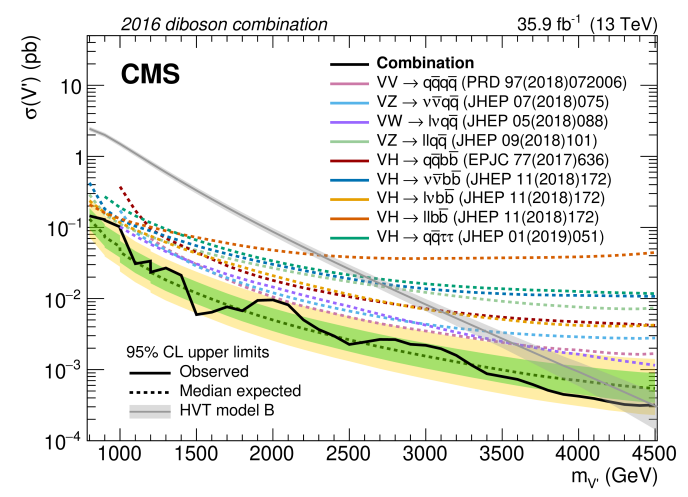

Figure 10: Observed and expected $95 \% \mathrm{CL}$ upper limits on cross sections as a function of the HVT triplet mass for the combination of all channels in the HVT model B. [18]

\section{References}

[1] CMS Collaboration, The CMS experiment at the CERN LHC, JINST 3 (2008) S08004

[2] CMS Collaboration, Search for a heavy Higgs boson decaying to a pair of W bosons in proton-proton collisions at $\sqrt{s}=13 T e V$, https: / / cds. cern. ch/record/2668687 
[3] CMS Collaboration, Search for a new scalar resonance decaying to a pair of Z bosons in proton-proton collisions at $\sqrt{s}=13 \mathrm{TeV}, \mathrm{JHEP} 06$ (2018) 127 [hep-ex/1804.01939]

[4] CMS Collaboration, Search for diboson resonances in the $2 \ell 2 v$ final state in proton-proton collisions at $\sqrt{s}=13 \mathrm{TeV}$, JHEP 03 (2018) 003 [hep-ex/1711.04370]

[5] CMS Collaboration, A multi-dimensional search for new heavy resonances decaying to boosted $W W$, WZ, or ZZ boson pairs in the dijet final state at $13 \mathrm{TeV}$, [hep-ex/1906.05977]

[6] CMS Collaboration, Search for a heavy resonance decaying to a pair of vector bosons in the lepton plus merged jet final state at $\sqrt{s}=13$ TeV, JHEP 05 (2018) 088 [hep-ex/1802 . 09407]

[7] CMS Collaboration, Search for a heavy resonance decaying into a $Z$ boson and a vector boson in the $\nu \bar{v} q \bar{q}$ final state, JHEP 07 (2018) 075 [hep-ex/1803 . 03838]

[8] CMS Collaboration, Search for a heavy resonance decaying into a $Z$ boson and a $Z$ or $W$ boson in $2 \ell 2 q$ final states at $\sqrt{s}=13$ TeV, JHEP 09 (2018) 101 [hep-ex/1803.10093]

[9] CMS Collaboration, Search for resonances decaying to a pair of Higgs bosons in the b $\bar{b} q \bar{q}^{\prime} \ell v$ final state in proton-proton collisions at $\sqrt{s}=13 \mathrm{TeV}$, [hep-ex/1904.04193]

[10] CMS Collaboration, Search for a massive resonance decaying to a pair of Higgs bosons in the four $b$ quark final state in proton-proton collisions at $\sqrt{s}=13 \mathrm{TeV}$, Phys. Lett. B 781 (2018) 244 [hep-ex/1710.04960]

[11] CMS Collaboration, Search for production of Higgs boson pairs in the four b quark final state using large-area jets in proton-proton collisions at $\sqrt{s}=13 \mathrm{TeV}$, JHEP 01 (2019) 040 [hep-ex/1808.01473]

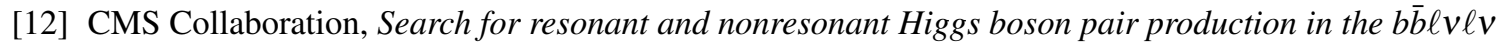
final state in proton-proton collisions at $\sqrt{s}=13 \mathrm{TeV}$, JHEP 01 (2018) 054 [hep-ex/1708.04188]

[13] CMS Collaboration, Search for Higgs boson pair production in the $\gamma \gamma b \bar{b}$ final state in pp collisions at $\sqrt{s}=13$ TeV, Phys. Lett. B 788 (2018) 7 [hep-ex/1806.00408]

[14] CMS Collaboration, Search for resonant pair production of Higgs bosons decaying to bottom quark-antiquark pairs in proton-proton collisions at $13 \mathrm{TeV}$, JHEP 08 (2018) 152 [hep-ex/1806.03548]

[15] CMS Collaboration, Search for heavy resonances that decay into a vector boson and a Higgs boson in hadronic final states at sqrt(s) = 13 TeV, EPJC 77 (2017) 636 [hep-ex/1707 . 01303]

[16] CMS Collaboration, Search for heavy resonances decaying into a vector boson and a Higgs boson in final states with charged leptons, neutrinos and b quarks at $\sqrt{s}=13 \mathrm{TeV}$, JHEP 11 (2018) 172 [hep-ex/1807.02826]

[17] CMS Collaboration, Search for heavy resonances decaying into two Higgs bosons or into a Higgs boson and $a \mathrm{~W}$ or $Z$ boson in proton-proton collisions at $13 \mathrm{TeV}, \mathrm{JHEP} 01$ (2019) 051 [hep-ex/1808.01365]

[18] CMS Collaboration, Combination of CMS searches for heavy resonances decaying to pairs of bosons or leptons, Phys. Lett. B 798 (2019) 134952 [hep-ex/1906. 00057 ]

[19] M. Cacciari, G. P. Salam, and G. Soyez, The anti- $k_{t}$ jet clustering algorithm, JHEP 04 (2008) 063 [hep-ph/0802.1189] 
[20] M. Dasgupta, A. Fregoso, S. Marzani, and G. P. Salam, Towards an understanding of jet substructure, JHEP 09 (2013) 029 [hep-ph/1307 . 0007]

[21] J. Thaler and K. Van Tilburg, Identifying Boosted Objects with N-subjettiness, JHEP 03 (2011) 015 [hep-ph/1011.2268]

[22] Y. Gao et al., Spin determination of single-produced resonances at hadron colliders, Phys. Rev. D 81 (2010) 075022 [hep-ph/1001.3396]

[23] I. Anderson et al., Constraining anomalous HVV interactions at proton and lepton colliders, Phys. Rev. D 89 (2014) 035007

[24] M. Carena et al., MSSM Higgs Boson Searches at the LHC: Benchmark Scenarios after the Discovery of a Higgs-like Particle, EPJC 73 (2013) 2552 [hep-ph/1302 . 7033]

[25] J. Dolen et al., Thinking outside the ROCs: Designing Decorrelated Taggers (DDT) for jet substructure, JHEP 05 (2016) 156 [hep-ph/ 1603.00027$]$ 